\title{
Video Article \\ Rapid Fluorescence-based Characterization of Single Extracellular Vesicles in Human Blood with Nanoparticle-tracking Analysis
}

\author{
Andreas Weber ${ }^{1}$, Julia Christin Wehmeyer ${ }^{1}$, Vera Schmidt ${ }^{1}$, Artur Lichtenberg ${ }^{1}$, Payam Akhyari ${ }^{1}$ \\ ${ }^{1}$ Department of Cardiovascular Surgery, Medical Faculty, Heinrich-Heine-University
}

Correspondence to: Artur Lichtenberg at artur.lichtenberg@med.uni-duesseldorf.de

URL: https://www.jove.com/video/58731

DOI: doi:10.3791/58731

Keywords: Biochemistry, Issue 143, Exosomes, nanovesicles, particle tracking analysis, CD63, CD9, vimentin, serum

Date Published: 1/7/2019

Citation: Weber, A., Wehmeyer, J.C., Schmidt, V., Lichtenberg, A., Akhyari, P. Rapid Fluorescence-based Characterization of Single Extracellular Vesicles in Human Blood with Nanoparticle-tracking Analysis. J. Vis. Exp. (143), e58731, doi:10.3791/58731 (2019).

\section{Abstract}

Extracellular vesicles (EVs), including exosomes, are specialized membranous nano-sized vesicles found in bodily fluids that are constitutively released from many cell types and play a pivotal role in regulating cell-cell communication and a diverse range of biological processes. Many different methods for the characterization of EVs have been described. However, most of these methods have the disadvantage that the preparation and characterization of the samples are very time-consuming, or it is extremely difficult to analyze specific markers of interest due to their small size and due to the lack of discrete populations. While methods for analysis of EVs have been considerably improved over the last decade, there is still no standardized method for characterization of single EVs. Here, we demonstrate a semi-automated method for characterization of single EVs by fluorescence-based nanoparticle-tracking analysis. The protocol that is presented addresses the common problem of many researchers in this field and provides the complete workflow for rapid isolation of EVs and characterization with PKH67, a general cell membrane linker, as well as with specific surface markers such as CD63, CD9, vimentin, and lysosomal-associated membrane protein 1 (LAMP-1). The presented results show a high level of reproducibility, as confirmed by other methods, such as Western blotting. In the conducted experiments, we exclusively used EVs isolated from human serum samples, but this method is also suitable for plasma or other body fluids and can be adjusted for characterization of EVs from cell culture supernatants. Irrespective of the future progress of research on EV biology, the protocol that is presented here provides a rapid and reliable method for rapid characterization of single EVs with specific markers.

\section{Video Link}

The video component of this article can be found at https://www.jove.com/video/58731/

\section{Introduction}

Extracellular vesicles (EVs), including exosomes, are specialized membranous nano-sized vesicles (20-150 nm) containing certain combinations of lipids, adhesion and intercellular signaling molecules, as well as other functional cytosolic components like microRNA (miRNA) and mRNA, and play a pivotal role in regulating cell-cell communication ${ }^{1,2}$. EVs are released in their environment from many different cell types, e.g., endothelial cells, immune cells, and tumor cells, and can be detected in body fluids such as serum semen, urine, breast milk, saliva, or cerebrospinal fluid $^{3,4}$. Increasing numbers of studies highlight the diverse contribution of EVs as potential biomarkers for early diagnosis of several diseases and/or prediction of disease progression ${ }^{5,6}$. Exosomes are often described by the presence of molecules that they are specifically associated with, regardless of the cell type they derive from ${ }^{7}$. For example, exosomes contain different tetraspanins (CD9, CD63, CD81), major histocompatibility complex class I (MHC I) molecules, various transmembrane proteins, typical cytosolic proteins (tubulin and actin), molecules involved in multivesicular body (MVB) biogenesis (TSG101 and alix), heat shock proteins (HSP 70 and HSP 90), and proteins that participate in signal transduction (protein kinases).

Many different methods have been described for the characterization of EVs ${ }^{9}$. The most common and prevalent methods used for EV analysis are flow cytometry ${ }^{10}$, scanning electron microscopy (SEM), and transmission electron microscopy (TEM) ${ }^{11}$. The best-established and commonly used method for the biochemical characterization of EV content is Western blotting ${ }^{12,13}$. While SEM and TEM allow for the detection of EVs across the entire size spectrum, the very limited identification of specific surface proteins is a particular disadvantage of these methods. In contrast, flow cytometry is a powerful tool for identification of specific EV surface markers, but the threshold of this method limits the analysis to EVs with a size greater than $500 \mathrm{~nm}$. Hence, analysis of isolated EVs with detection of specific surface markers is currently not accessible through any of these three well-established methods. We previously described another highly sensitive method for visualization and analysis of EVs, nanoparticle-tracking analysis (NTA) ${ }^{14}$. Briefly, this method combines two different physical principles. First, particles scatter light when they are irradiated with a laser beam, and the second principle, known as Brownian motion, implies that diffusion of different particles in a liquid suspension is inversely proportional to their size. The semi-automated desktop nanoparticle analysis instrument for liquid samples consists of the particle tracking analyzer with a software-based analysis, where digital images of scattered light from single particles are recorded. The particles and the movement of the particles are detected by a laser scattering microscope with a video camera. The laser beam is oriented vertically, while the optical axis is horizontal and focused into the cell channel filled with the sample. The data provided by plots of scattered light spots and their speed of motion enable the determination of total particle count and size distribution. After irradiation by the laser, the particles scatter the light, which is recorded by a digital video camera via the microscope ${ }^{14}$. The advancement to our former method is the insertion of a 500 nm long 
wave-pass (LWP) cut-off filter between the laser (wavelength of $488 \mathrm{~nm}$ ) and the cell channel, which enables the direct analysis of fluorescencelabeled particles (Figure 1). Our protocol addresses the common demand of many researchers in this field for a fast characterization of single EVs, e.g., according to their parental origin. In this protocol, we describe the complete workflow for rapid isolation of EVs from human whole blood and fast characterization of specific markers by fluorescence-based nanoparticles-tracking analysis. EVs can be detected by staining with PKH67, a general cell membrane linker, as well as with specific exosomal markers, e.g., CD63, CD9, and vimentin. Our protocol is also suitable for EDTA and citrated plasma, as well as other body fluids and cell culture supernatants.

\section{Protocol}

The institutional ethical board of the University of Dusseldorf has approved the experiments presented in this work (Reference number: 3381 ).

\section{EV Isolation from Human Whole Blood}

1. Isolate EVs with exosome precipitation solution.

1. Collect $2 \mathrm{~mL}$ of human whole blood in serum-separating tubes (SST) via venipuncture and incubate the tube for $15 \mathrm{~min}$ at room temperature (RT) until coagulation is finished.

2. Centrifuge the SST at $1,700 \times g$ for $10 \mathrm{~min}$ at $\mathrm{RT}$ to separate cells from serum and transfer $1 \mathrm{~mL}$ of the serum to a $1.5 \mathrm{~mL}$ reaction tube. Centrifuge the platelet-rich plasma (PRP) at $3,000 \times \mathrm{g}$ for $15 \mathrm{~min}$ at $4{ }^{\circ} \mathrm{C}$ to remove platelets and transfer $100 \mu \mathrm{L}$ of the plateletpoor plasma (PPP) to a new $1.5 \mathrm{~mL}$ reaction tube.

3. Add $25 \mu \mathrm{L}$ of exosome precipitation solution (4 parts PPP, 1 part exosome precipitation solution) and vortex thoroughly. Incubate the sample for $30 \mathrm{~min}$ on ice. Keep the tube upright and do not rotate or mix the tube during the incubation period.

4. Centrifuge the sample at $1,500 \times g$ for $30 \mathrm{~min}$ at $4{ }^{\circ} \mathrm{C}$ to pellet the EVs. After centrifugation, the EVs appear as a beige or white pellet at the bottom of the vessel. Aspirate the supernatant and centrifuge the sample at $1,500 \times \mathrm{g}$ for $5 \mathrm{~min}$ at $4{ }^{\circ} \mathrm{C}$.

5. Remove all traces of fluid and re-suspend the pellet in $100 \mu \mathrm{L}$ of phosphate-buffered saline (PBS) by frequently pipetting up and down. Store the EV suspension at $-80^{\circ} \mathrm{C}$ when analysis is not performed immediately.

Note: When isolating EVs from plasma, fibrinogen and fibrin can impede efficient recovery and resuspension is heavier and takes more time.

2. Isolate EVs with ultracentrifugation.

1. Take the pre-cooled rotor (TLA-55 fixed-angled) from the fridge and cool down the ultracentrifuge before use.

2. Transfer $1.25 \mathrm{~mL}$ of PPP (prepared in step 1.1) to a suitable $1.5 \mathrm{~mL}$ ultracentrifugation tube with cap and centrifuge the sample at $110,000 \times g$ for $90 \mathrm{~min}$ at $4{ }^{\circ} \mathrm{C}$. Make sure that the rotor load is balanced before starting.

3. Decant the supernatant and place tube upside-down on a paper towel for 2 min. Re-suspend the pellet in $500 \mu \mathrm{L}$ of PBS and centrifuge the sample at $110,000 \times g$ for $90 \mathrm{~min}$ at $4{ }^{\circ} \mathrm{C}$.

4. Aspirate the supernatant and re-suspend the pellet in $50 \mu \mathrm{L}$ of PBS.

Note: Use only rotors and accessories designed for the ultracentrifuge that is in use. Pretest the tubes in the rotor by using water because the strength of the tubes can vary between lots.

\section{Staining of the Samples}

1. Stain samples with PKH67.

1. Prepare the staining solution by adding $1 \mu \mathrm{L}$ of the $\mathrm{PKH} 67$ ethanolic dye solution to $50 \mu \mathrm{L}$ of diluent $\mathrm{C}$ (included in the kit) in a $1.5 \mathrm{~mL}$ reaction tube and mix thoroughly.

2. Transfer $10 \mu \mathrm{L}$ of the staining solution to $20 \mu \mathrm{L}$ of the EV suspension (prepared in step 1.1) to the reaction tube, mix thoroughly and incubate for 5 min at RT in the dark.

3. Dilute $50 \mu \mathrm{L}$ of the stained EV suspension with $2.5 \mathrm{~mL}$ distilled water in a $15 \mathrm{~mL}$ reaction tube, mix thoroughly, and use this final suspension for particle measurement.

Note: It is crucial to use water as diluent for the EV suspension, because other diluents (e.g., PBS) can impair the measurement. When using stored EV-suspension, thaw the samples on ice and mix thoroughly before staining.

2. Stain samples with specific antibodies.

1. Dilute $10-20 \mu \mathrm{L}$ of the EV suspension (prepared in step 1.1) with $50 \mu \mathrm{L}$ of distilled water in a $1.5 \mathrm{~mL}$ reaction tube and mix thoroughly.

2. Add 2.5-5 $\mu \mathrm{L}$ of the specific antibody (Table 1) to the reaction tube, mix thoroughly, and incubate for $30 \mathrm{~min}$ at RT in the dark.

3. Transfer $50 \mu \mathrm{L}$ of the stained EV suspension to $2.5-10 \mathrm{~mL}$ of distilled water (Table 1) in a $15 \mathrm{~mL}$ reaction tube, mix thoroughly, and use this final suspension for particle measurement.

Note: Under some circumstances, the concentration of the used antibody must be adjusted (Table 1).

\section{Processing of the Samples}

Note: The fundamentals of this method were extensively described previously and below the protocol focuses on the specific steps needed for analysis of fluorescence-labeled $\mathrm{EVs}^{14}$.

1. Perform the startup procedure.

1. Push the fluorescence filter into the optical path of the microscope and camera. Start the program and follow the instructions on the screen for automated implementation. 
2. Select the correct cell number (Z158 C1149 Fluor) in the "Cell Check" tab (cell definition) for fluorescence measurement. Select the reference position for the optics to make sure that laser and microscope are in a common focus (laser and microscope move to this position automatically).

3. Flush the cell channel with a syringe filled with $10 \mathrm{~mL}$ of distilled water. Ensure that the measurement cell is free of air bubbles and do not inject air bubbles into the system.

4. Prepare a calibration suspension containing uniform $200 \mathrm{~nm}$ sized fluorescence-labelled polystyrene particles that have carboxylate groups on their surface. Dilute $10 \mu \mathrm{L}$ of the particles with $990 \mu \mathrm{L}$ distilled water. Then dilute $10 \mu \mathrm{L}$ of this particle solution in a $15 \mathrm{~mL}$ tube with $10 \mathrm{~mL}$ of distilled water to obtain the required concentration.

5. Inject $2.5 \mathrm{~mL}$ of the diluted particle solution in the cell channel and click "Optimize focus" to adjust the camera.

2. Measure the sample.

1. Flush the cell channel multiple times with a syringe filled with $10 \mathrm{~mL}$ of distilled water prior to each sample measurement. Inject the stained EV suspension (prepared in step 2) into the cell channel.

2. Adjust the following main camera parameters in the "Cell Check" tab in the software as needed (Table 2). Use the reference position or position 0.41193 to adjust the parameters.

1. For sensitivity, find the optimal sensitivity range by clicking on the button "Number of Particles vs. Sensitivity" to display a curve of measured particles per screen for different sensitivity levels.

Note:

2. For shutter, adjust the period of time that the camera allows light to pass for a determined interval.

3. For post-acquisition parameters, choose a minimum brightness of 20 , a minimum size of $20 \mathrm{~nm}$, and a maximum size of $500 \mathrm{~nm}$ for measurement.

3. Note the number of detected particles counted in the field of vision from the display. The scattering bar must be in the green to orange range (50-300 particles). If the scattering bar is red, the scatter will fuse individual particles and they are counted as one single particle, thus leading to false results. In such a case, further dilute the sample to avoid overlapping of the particles or lower the sensitivity.

4. Click on "Check particle drift at $0 \mathrm{~V}$ " in the "Cell Check" tab before starting the measurement. If the drift is higher than $5 \mu \mathrm{m} / \mathrm{s}$, wait until the sample stops flowing to continue the measurement.

Note: If the drift is too high at the beginning of the measurement, the repeated measurements can differ and sophisticate the results. Due to the underlying principles of NTA, a relevant drift may have an impact in the determined particle size, as calculated by the software.

5. Click on "Run Video Acquisition" in the "Measurement" tab. Select the number of experiments (3-5) and the time delay between them (0 $\min )$.

6. Define the number of (individual subvolume-) positions (11) and the number of (measurement) cycles (10) at each measurement position, where particles should be analyzed

7. Select a folder, create a new file name, and click "OK" to start the measurement.

\section{Interpretation of the Results}

1. View the results and parameters on the "Analysis" tab after the measurement. Check the following parameters after the analysis before cleaning the cell channel: average number of particles per position, total number of traced particles and particle concentration, the distribution width of the particles (x10, x50, and x90 values), value of mean and standard deviation. Repeat the measurement of the sample if required. Note: The results are also saved as a .pdf or .txt file.

2. Click on "Analysis" tab to view the graph calculated after the measurement, which shows the distribution of the detected particles by size. Click on "Display" in the "Analysis" tab and use the icons to adjust and change the graph settings for particular requirements.

\section{Validation via EV Detection by Western Blotting}

1. Dissolve the EV pellet (prepared in step 1) in RIPA lysis and extraction buffer, pipette thoroughly and incubate on ice for 30 min. Centrifuge the sample at $8,000 \times g$ for $10 \mathrm{~min}$ at $4{ }^{\circ} \mathrm{C}$ to clarify the lysate and transfer the supernatant to a new tube.

2. Measure the total protein by a Lowry protein assay kit. Dilute $1 \mu \mathrm{L}$ of the isolated EV suspension with $49 \mu \mathrm{L}$ of RIPA buffer and use $5 \mu \mathrm{L}$ in triplicates for analysis. Dilute the EV suspension with $2 x$ Laemmli loading buffer to a final concentration of $2 \mu \mathrm{g} / \mu \mathrm{L}$ and heat for $10 \mathrm{~min}$ at 95 ${ }^{\circ} \mathrm{C}$

3. Load $20 \mu \mathrm{g}$ of protein per well. Separate and transfer the proteins by polyacrylamide gel electrophoresis and tank blotting according to standard protocols.

4. Block the membrane $(0.2 \mu \mathrm{m}$ polyvinylidene difluoride) with bovine milk powder (5\%) for $1 \mathrm{~h}$ at RT and incubate the membrane with specific primary antibodies (CD9, CD63, and vimentin) overnight at $4{ }^{\circ} \mathrm{C}$.

Note: The primary antibodies are used at a 1:1,000 dilution.

5. Wash the membrane with TBST $3 \times 5$ min and incubate the membrane with horseradish peroxidase (HRP)-conjugated secondary antibody (1:20,000 in TBST) for 60 min at RT.

6. Wash the membrane with TBST $3 \times 5$ min and detect the proteins by chemiluminescence with a high sensitivity substrate solution on an imaging system.

Note: Because CD63 antigen is extensively and variably glycosylated, the molecular weight can vary, and bands can appear between $40-65$ $\mathrm{kDa}$. 


\section{Representative Results}

EVs were isolated from whole blood and characterized by nanoparticle tracking analysis with fluorescing reagents. The optimal sensitivity for measurement of unstained particles was identified to range at $70 \%$ during our experiments. The fluorescent beads used for adjustment and calibration of the measurement showed an optimum setting at a sensitivity of $85 \%$ (Figure 2A). Between a sensitivity of $70 \%$ and $90 \%$, the number of detected particles increased rapidly, while further increasing the sensitivity can lead to a deterioration of the particle size distribution where the number of particles is re-dropping. The settings of the camera displayed a sharp picture (Figure 2B) and repeated measurements showed low standard deviation (Figure 2C). Insofar, the protocol for processing the samples of EVs was adjusted so that all measurements could be conducted with the same settings (Table 2). The distribution width is defined by three values on the $x$-axis, the $x 10$, $x 50$, and $x 90$. The $x 50$, or median particle size is the diameter at which half of the population lies below this value. Similarly, the $x 10$ and $x 90$ indicate the diameter at which $10 \%$ and $90 \%$ of the detected particles are under the reported size. Staining with PKH67 cell linker kit, including a fluorescent cell linker that incorporates a green fluorescent dye with long aliphatic tails into lipid regions of the cell membrane, demonstrated a strong correlation between the sensitivity and the number of particles measured (Figure 3A). PKH67 is often used for proliferation monitoring but has also proven useful for monitoring exosome or liposome uptake as well as for in vivo cell trafficking. Due to the non-specific labeling of $\mathrm{PKH67}$, a wide variety of EVs can be labeled and detected. The distribution of the particles was in a range between $266 \mathrm{~nm}(\mathrm{x} 10)$ and 1946 $\mathrm{nm}$ (x90) with a peak maximum at $857 \mathrm{~nm}$ (x50) and with a low standard deviation between the measurements (28.1 nm). LAMP-1, also known as lysosome-associated membrane glycoprotein 1, and CD107a reside primarily across lysosomal membranes. After staining with an Alexa Fluor 488 labeled specific antibody against LAMP-1, the distribution of the particles ranges from $220 \mathrm{~nm}(\mathrm{x} 10)$ to $1145 \mathrm{~nm}$ (x90) with a peak maximum at $541 \mathrm{~nm}$ (x50) and a standard deviation of $11.7 \mathrm{~nm}$ (Figure 3B). For characterization of EVs, we used Alexa Fluor 488 labeled antibodies against common exosomal markers and confirmed our findings by Western blotting. After staining with Alexa Fluor 488-labeled CD9antibody, the distribution of the particles ranges from $251 \mathrm{~nm}(\mathrm{x} 10)$ to $1139 \mathrm{~nm}$ (x90) with a peak maximum at $548 \mathrm{~nm}$ and a second minor peak at approximately $25 \mathrm{~nm}$ (Figure 4A). Staining with Alexa Fluor 488 labeled CD63 (Figure 4B) and vimentin (Figure 4C) yielded similar results. Western blotting analysis substantiated our positive result for antibodies used here. Repeated measurements showed reproducible results for all antibodies used in this report. As controls, we stained vesicle-free water with respective antibodies (Figure 5A), where PKH67 and LAMP1 antibodies virtually detected no EV up to a sensitivity close to $100 \%$. Using the example of vimentin, high sensitivity increased the number of emerging artefacts, even when the sample is essentially free of particles. If measurement is started when the drift is yet too high (> $5 \mu \mathrm{m} / \mathrm{s})$, the individual repetitions distinctly deviate among themselves (Figure 5B). As represented with three different antibodies, it is crucial that the drift is as minimal as possible before starting the measurement. According to our experience, using fluorescein isothiocyanate (FITC) as fluorochrome results in measurements that are not accurate and reproducible because FITC is prone to rapid photo bleaching (Figure 5C). Therefore, we recommend using exclusively Alexa Fluor 488 labeled antibodies for EV characterization. In this protocol, EVs were isolated by a polymerbased exosome precipitation solution containing polyethylene glycol. To ensure that our results are not falsified by the applied isolation method, we characterized EVs after isolation with ultracentrifugation. As represented with PKH67 and two different antibodies (CD63 and LAMP-1), the results of our applied isolation with exosome precipitation solution (Figure 6A) are comparable with EVs isolated via ultracentrifugation (Figure 6B). Unfortunately, because of the poor yield of EVs after ultracentrifugation, the initial set of serum for isolation must be distinctly higher compared to isolation with exosome precipitation solution.

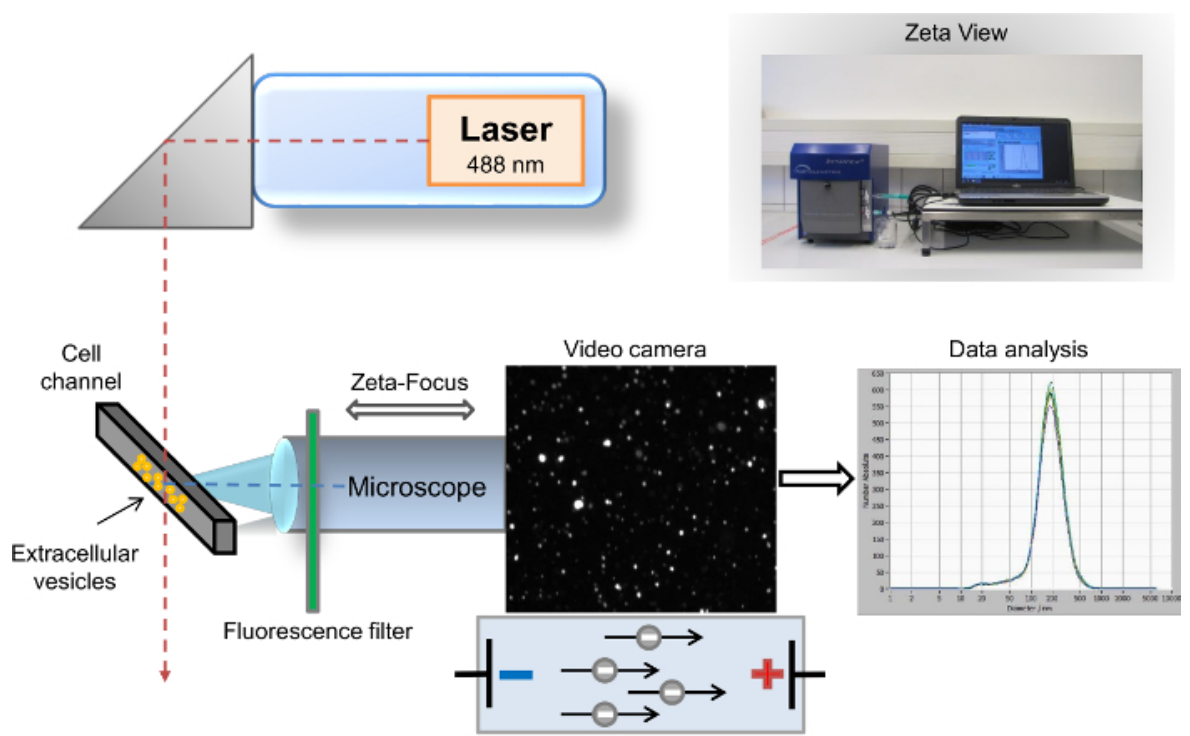

Figure 1: Schematic setup of the nanoparticle tracking analysis. The microscope/video axis and laser beam are orientated orthogonally to each other, crossing at the cell channel cross section. A fluorescence filter is placed between the cell channel and the microscope. Light scattered by the particles is displayed in the "live-view" window of the software. After acquisition, the results are displayed as a size distribution curve coordinate system. Please click here to view a larger version of this figure. 


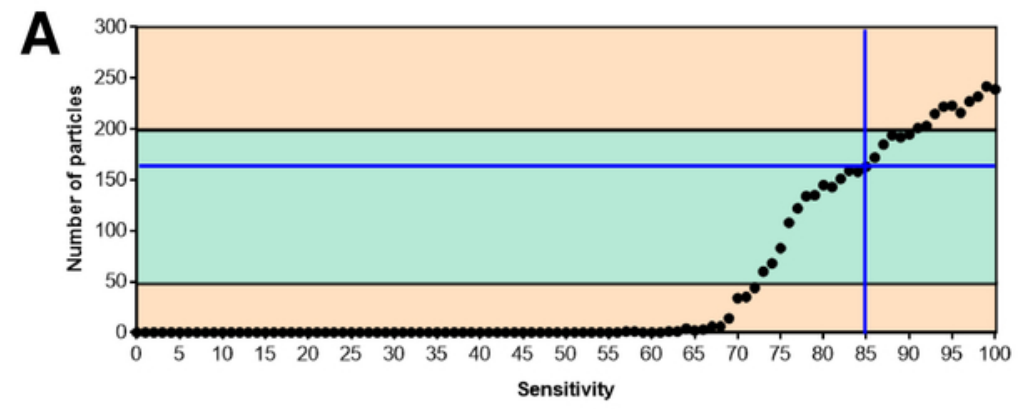

B

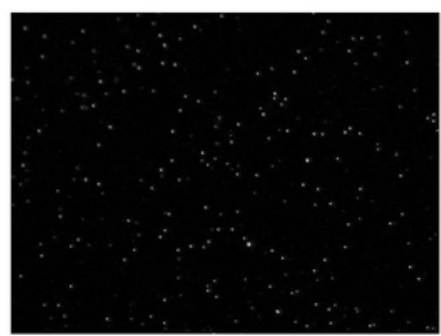

C

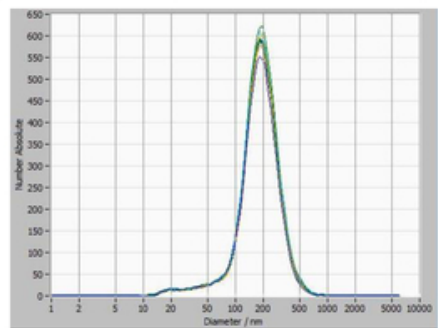

Figure 2: Calibration with fluorescence labeled beads. (A) The number of particles vs. sensitivity curve displays the particles in one position at one point in time during an automatic sensitivity scan. (B) Visualization of particles on the live view screen. (C) Particle size distribution after repeated measurements. Please click here to view a larger version of this figure.

A

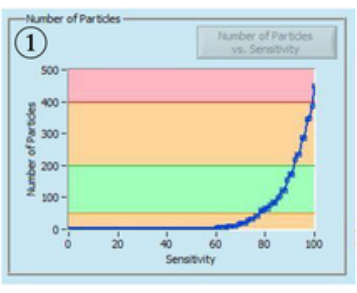

\section{PKH67}

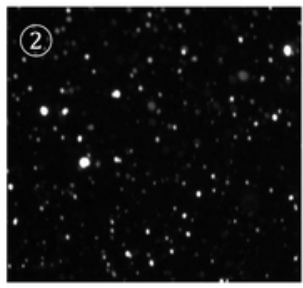

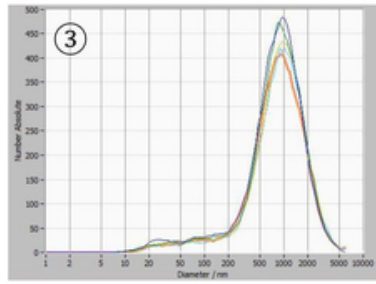

\section{B}

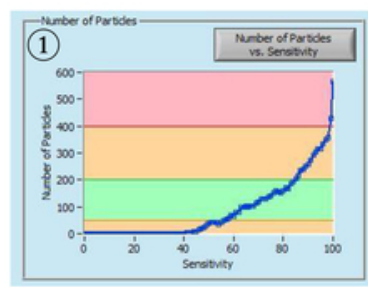

LAMP-1
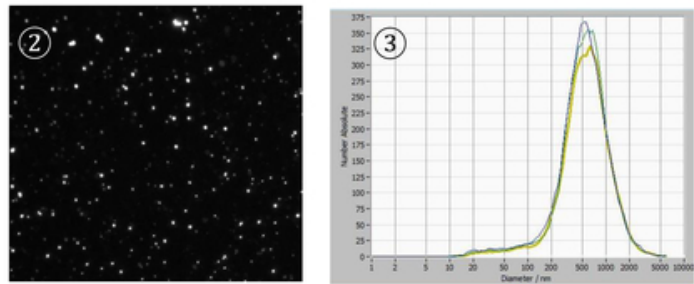

Figure 3: Representative results after staining with PKH67 and LAMP-1. Number of particles vs. sensitivity curve (1), visualization of particles on the live view screen (2), and particle size distribution (3) after staining with PKH67 (A) and LAMP-1 (B). A similar size distribution of particles is observed, while changes in the sensitivity setting lead to a similar but yet not entirely identical change of detected particles. Please click here to view a larger version of this figure. 

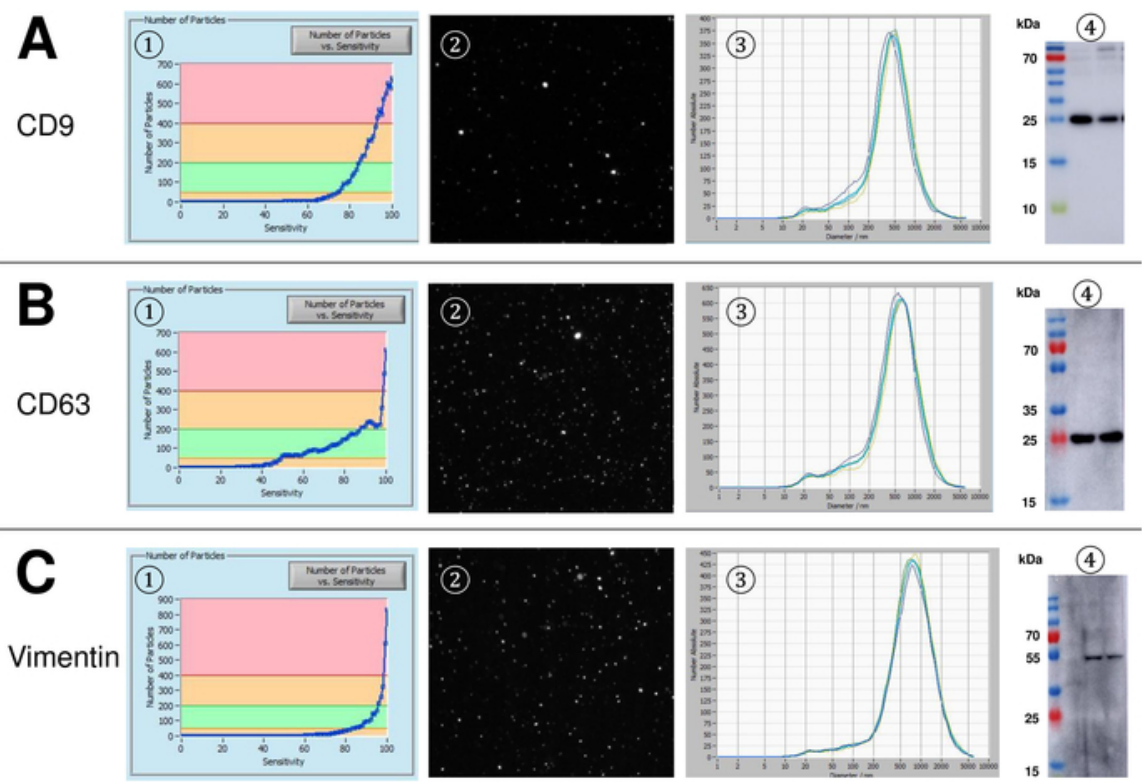

Figure 4: Representative results after staining with Alexa Fluor 488 labeled CD9, CD63, and vimentin. Number of particles vs. sensitivity curve (1), visualization of particles on the live view screen (2), particle size distribution (3), and representative Western blots of two different EV suspensions (4) for CD9 (24-27 kDa, A), CD63 (26 kDa, B), and vimentin (54 kDa, C). Late occurrence of particle signals along the increasing sensitivity (x-axis) correlates with lower signal intensity in the Western blot analysis, confirming the lower amount of the respective particle surface marker (e.g., vimentin vs. CD63). Note the almost identical curves of representative measurements for all analyzed markers indicating high reproducibility (3). Please click here to view a larger version of this figure.
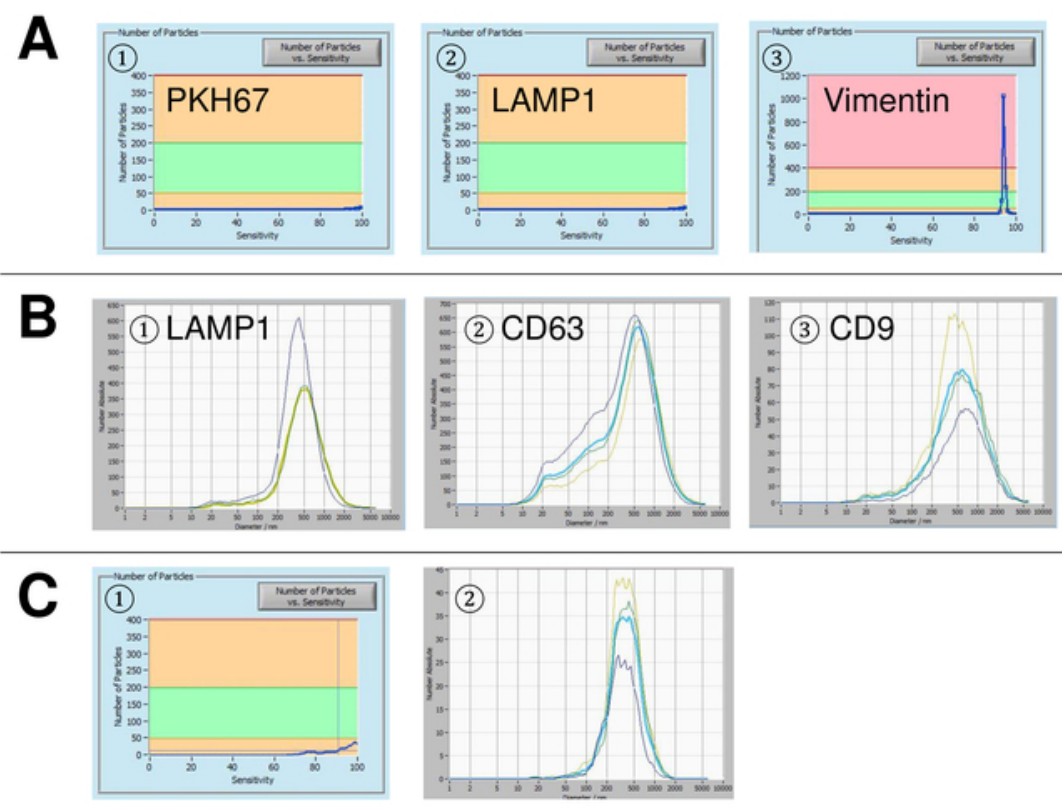

Figure 5: Representative results for used controls and possible error sources. (A) Vesicle-free water stained with PKH67 (1), LAMP-1 (2), and vimentin (3) as controls. (B) Representative particle size distributions after staining with LAMP-1 (1), CD63 (2), and CD9 (3), and measurements performed when suspension drift is yet too high. (C) Number of particles vs. sensitivity curve (1) and particle size distribution (2) after staining with FITC-labelled CD63 antibody. A clear bleaching effect is observed after each measurement, resulting in progressively lower particle numbers. Please click here to view a larger version of this figure. 


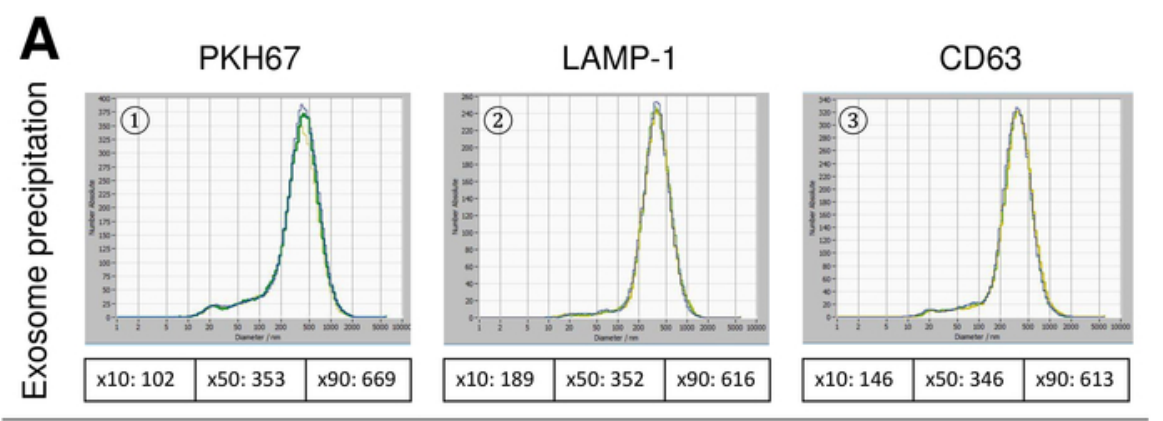

B
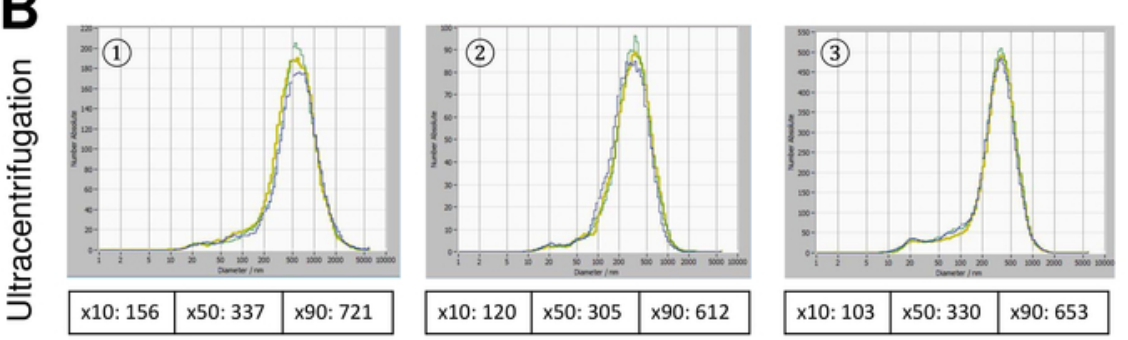

Figure 6: Comparison of different isolation methods for EVs. Particle size distribution of EVs isolated with exosome precipitation solution (A) and ultracentrifugation (B) after staining with PKH67 (1), LAMP-1 (2), and CD63 (3). Please click here to view a larger version of this figure.

\begin{tabular}{|l|l|l|l|l|}
\hline & LAMP-1 & CD9 & CD63 & Vimentin \\
\hline Antibody $(\boldsymbol{\mu L})$ & 5 & $2.5-5$ & $2.5-5$ & 5 \\
\hline EV suspension $(\boldsymbol{\mu L})$ & 10 & 20 & 10 & 10 \\
\hline $\mathrm{H}_{2} \mathrm{O}(\boldsymbol{\mu L})$ for staining & 50 & 50 & 50 & 50 \\
\hline Final volume $(\mathrm{mL})$ & $5-10$ & $2.5-5$ & 5 & 10 \\
\hline
\end{tabular}

Table 1: List of used antibodies and dilution range of samples.

\begin{tabular}{|l|l|}
\hline Acquisition parameters & \\
\hline Sensitivity $(\%)$ & 85 \\
\hline Shutter & 70 \\
\hline Min. Brightness & 20 \\
\hline Max. Size $(\mathrm{nm})$ & 500 \\
\hline Min. Size $(\mathrm{nm})$ & 20 \\
\hline Polarity & Negative \\
\hline Voltage & Off \\
\hline Particle drift at 0 V $(\mu \mathrm{m} / \mathrm{s})$ & $<5$ \\
\hline Positions & 11 \\
\hline Cycles & 10 \\
\hline Multiple acquisitions & $3-5$ \\
\hline Time delay (min) & 0 \\
\hline
\end{tabular}

Table 2: Acquisition parameters for nanoparticle tracking analysis.

\section{Discussion}

We demonstrate a detailed protocol for isolation of EVs from whole blood and fast characterization of specific surface markers with fluorescencebased nanoparticles tracking analysis. In the conducted experiments, we exclusively used EVs isolated from serum samples, but this method is also suitable for ethylenediaminetetraacetic acid (EDTA) and citrated plasma and can also be expanded to other bodily fluids such as urine, breast milk, saliva, cerebrospinal fluid, and semen. Moreover, this protocol can be adjusted for characterization of EVs from cell culture supernatants. In this protocol, the EV suspension was generated from $100 \mu \mathrm{L}$ of serum using an exosome precipitation reagent, which contains a proprietary polymer that gently precipitates exosomes and EVs according to a corpuscular size ranging from $30 \mathrm{~nm}$ to $200 \mathrm{~nm}$, whereby $10-20$ 
$\mu \mathrm{L}$ of EVs were appointed for characterization of each surface marker. Unfortunately, the isolation step is inevitable, because the high amount of protein in serum samples (e.g., albumin and globulin) interferes with the antibody staining procedure and results in high level of background and sophisticated findings. Furthermore, based on the biological availability of the exosomes in the samples, the amount of the employed EV suspension as well as the dilution before processing must be adjusted for other source materials. To compare multiple samples, a standardized approach for the dilution of the samples as well as consistent acquisition parameters (sensitivity, shutter, etc.) is necessary. Another important point is that the measurements are not started until the drift is low (in our hands, $<5 \mu \mathrm{m} / \mathrm{s}$ ). If the drift was too high, repeated measurements of the sample yielded high standard deviation among themselves, but with a low drift, the resulting data were highly consistent and confirmed a high level of reproducibility. It is important that the selected antibodies have an appropriate fluorochrome. Antibodies must be conjugated with Alexa Fluor 488, because FITC has a high rate of photo-bleaching. Possibly more stable fluophores will certainly lead to increased assay stability in the future. Normally, many researchers use PBS as a diluent for EVs. For this protocol, it is crucial to use distilled water as a diluent for the EV suspensions. When EVs are labeled with fluorescing dyes, the high osmolality and ion concentration of other diluents, such as PBS, can interfere with the measurement and lead to altered results.

While methods for analysis of EVs have been considerably improved over the last decade, there is still no standardized method for isolation and characterization of EVs. The major disadvantage of flow cytometry, where EVs are often bound to beads to provide a larger surface, is that many EVs dock onto the surface to provide a strong and detectable signal ${ }^{10}$. SEM and TEM have the disadvantage that the preparation of samples is time-consuming and EVs can only be distinguished by their size and morphology ${ }^{11}$. Up to date, the best-established and commonly used method for qualitative (i.e., biochemical) EV characterization is Western blotting, where proteins can be analyzed with specific antibodies ${ }^{12,13}$. However, the disadvantages of all these methods lie in the inability to analyze single EVs for specific surface markers. Furthermore, the long processing times and long washing/isolation procedures used by many of the current protocols involve labor-intensive steps, making them not suitable for high sample throughput and characterization of single EVs. Our protocol provides a complete workflow for quick isolation and characterization of single EVs with specific surface markers such as CD63, CD9, vimentin, and CD107a, and can be expanded for a broad spectrum of other surface markers to determine the origin of released EVs. Because of a permanent technical advancement of the NTA device, we confirmed our findings in cooperation with the manufacturer with the newest analyzer. Irrespective of the future progress of research on EV biology, particularly concerning exosomes, the protocol that is presented here will provide a rapid and reliable method for characterization of single EVs with specific markers. Because aggregation of EVs during the isolation and staining procedure is so far unavoidable, future research should focus on developing methods to prevent EV aggregation and enable an accurate size determination of fluorescent-labeled EVs.

\section{Disclosures}

The authors have nothing to disclose.

\section{Acknowledgements}

The authors thank Particle Metrix $\mathrm{GmbH}$ for partially covering the publication costs of this work.

\section{References}

1. Ibrahim, A., \& Marban, E. Exosomes: Fundamental Biology and Roles in Cardiovascular Physiology. Annual Review of Physiology. 78, 67-83 (2016).

2. Su, S. A. et al. Emerging role of exosome-mediated intercellular communication in vascular remodeling. Oncotarget. 8 (15), $25700-25712$ (2017).

3. Lasser, C. et al. Human saliva, plasma and breast milk exosomes contain RNA: uptake by macrophages. Journal of Translational Medicine. 9, 9 (2011).

4. $\mathrm{Li}, \mathrm{M}$. et al. Analysis of the RNA content of the exosomes derived from blood serum and urine and its potential as biomarkers. Philosophical Transactions of The Royal Society B Biological Sciences. 369 (1652) (2014).

5. Lin, J. et al. Exosomes: novel biomarkers for clinical diagnosis. The Scientific World Journal. 2015, 657086 (2015).

6. Properzi, F., Logozzi, M., \& Fais, S. Exosomes: the future of biomarkers in medicine. Biomarkers in Medicine. 7 (5), $769-778$ (2013).

7. Thery, C., Zitvogel, L., \& Amigorena, S. Exosomes: composition, biogenesis and function. Nature Reviews Immunology. 2 (8), $569-579$ (2002).

8. De Toro, J., Herschlik, L., Waldner, C., \& Mongini, C. Emerging roles of exosomes in normal and pathological conditions: new insights for diagnosis and therapeutic applications. Frontiers in Immunology. 6, 203 (2015).

9. Thery, C., Amigorena, S., Raposo, G., \& Clayton, A. Isolation and characterization of exosomes from cell culture supernatants and biological fluids. Current Protocols in Cell Biology. Chapter 3, Unit 3, 22 (2006).

10. Inglis, H., Norris, P., \& Danesh, A. Techniques for the analysis of extracellular vesicles using flow cytometry. Journal of Visualized Experiments. (97) (2015)

11. Wu, Y., Deng, W., \& Klinke, D. J., 2nd. Exosomes: improved methods to characterize their morphology, RNA content, and surface protein biomarkers. Analyst. 140 (19), 6631-6642 (2015).

12. Bianco, N. R., Kim, S. H., Morelli, A. E., \& Robbins, P. D. Modulation of the immune response using dendritic cell-derived exosomes. Methods in Molecular Biology. 380, 443-455 (2007).

13. Conde-Vancells, J. et al. Characterization and comprehensive proteome profiling of exosomes secreted by hepatocytes. Journal of Proteome Research. 7 (12), 5157-5166 (2008).

14. Mehdiani, A. et al. An innovative method for exosome quantification and size measurement. Journal of Visualized Experiments. (95), 50974 (2015). 